\title{
Review of: "Grip strength as a predictor of depressive symptoms among vulnerable elderly Europeans with musculoskeletal conditions"
}

Sang Yeoup Lee

Potential competing interests: The author(s) declared that no potential competing interests exist.

The prevalence of depressive symptoms in this study population was approximately $30 \%$.

All subjects in this study had MSK conditions, so the prevalence was as high as expected. So then, rather than measuring grip strength to identify who has depressive symptoms, wouldn't it be better to ask if everyone with MSK conditions has depressive symptoms directly?

It was noted in the introduction that depression and pain share biological pathways and neurotransmitters. It should appear equally in men and women. However, the phenomenon appeared in men in one study and only in women in another study. Therefore, it seems that there are still more parts to be revealed. 